\title{
FUENTES ORALES E IDENTIDADES PROFESIONALES: \\ LAS MÉDICAS ESPAÑOLAS EN LA SEGUNDA MITAD DEL SIGLO XX
}

\author{
Teresa Ortiz Gómez \\ Catedrática de Historia de la Ciencia, Universidad de Granada
}

\section{RESUMEN}

En este trabajo se analiza interés y el significado de las fuentes orales para la elaboración de biografías colectivas y relatos prosopográficos y para la historia de las profesiones y la asistencia sanitaria. Se ejemplifica con el estudio de la experiencia profesional de las médicas españolas de la segunda mitad del siglo XX a partir de entrevistas en profundidad.

PALABRAS CLAVE: Historia oral, prosopografía, mujeres y medicina, género y profesión médica, identidades y género, asociacionismo médico.

\section{SUMMARY}

This paper analyses the importance and significance of oral sources for the production of collective biographies and prosopographic studies and for the history of the health professions and healthcare. As an example, it contains a study of the professional experience of female Spanish doctors in the second half of the 20th century based on in-depth interviews.

KEY WORDS: Oral history, prosopography, women physicians, gender and medical profession, gender identities, medical societies.

La prosopografía, o historia de grupos de personas dentro de un contexto histórico, social y político, persigue no tanto biografiar al grupo en su conjunto como poner en relación las biografías individuales de sus componentes. ${ }^{1}$ De hecho, bajo la denominación grupos, hay que incluir tanto a aquellos que existieron como tales en el pasado, manteniendo una mínima estructura organizativa y una conciencia de tales, como a grupos creados a posteriori con un

1 Una revisión reciente sobre el tema en KEATs-RoHAN, K.S.B. (2004) Progress or perversion? Current issues in prosopography: An introduction.

http://www.linacre.ox.ac.uk/research/prosop/prosopo.stm (consultada en junio de 2004). 
objetivo historiográfico y de acuerdo con el criterio que guía la investigación. En este trabajo, las médicas españolas en la segunda mitad del siglo XX son consideradas como un conjunto de profesionales que comparten unas circunstancias sociales y laborales determinadas, y cambiantes en el tiempo, dentro de un doble sistema de género que organiza la vida social, en general, y la actividad sanitaria en particular.

El uso, en exclusividad o en combinación con otras fuentes, de relatos autobiográficos implica incorporar la subjetividad, o el hecho histórico subjetivo, en la construcción de la historia de la medicina y, más concretamente, de la historia de la práctica médica. Contemplar la subjetividad no es una cuestión estrictamente nueva, acostumbrados como estamos en la disciplina a manejar opiniones e ideas de médicos y de legos recogidas en artículos periodísticos, conferencias, textos divulgativos, epistolarios e incluso diarios y memorias personales. El cambio principal que propone la historiografía reciente afecta al nuevo carácter epistémico que se otorga a la subjetividad: el hecho, por una parte, de que quien investiga pueda generar e intervenir en la creación de la propia fuente de estudio, y el hecho, también, de que se busque y se trate de analizar la subjetividad en si misma, más que someterla a una cuarentena crítica para evaluar en qué medida lo dicho y oído responde o no a «la verdad», o a la realidad.

Lo voy a ejemplificar con el caso de la experiencia profesional de las médicas españolas de la segunda mitad del siglo XX, y más concretamente en lo relacionado con un proyecto de asociacionismo profesional que ellas pusieron en marcha en 1965. Previamente, revisaré el interés y el significado de las fuentes orales para la historia de la medicina y para la elaboración de biografías médicas colectivas contemporáneas.

\section{FUENTES ORALES EN LA HISTORIA DE LA MEDICINA Y DE LA CIENCIA}

El creciente interés historiográfico por el estudio de los discursos y prácticas no hegemónicas, o no dominantes, las propuestas de integrar voces diversas en la construcción de la historia, así como el renovado y revisado interés por la microhistoria, o historia de ejemplos localizados y detallados de estudios de casos, están llamando la atención hacia las fuentes orales, especialmente entrevistas en profundidad, entrevistas semi-estructuradas e historias de vida. Se trata de un recurso muy poco utilizado en la historia de la medicina española que, sin embargo, podría formar parte de las técnicas de investi- 
gación de la mayor parte de los proyectos de historia de la medicina contemporánea actualmente en marcha ${ }^{2}$.

El recurso a las fuentes orales no es nuevo en la bibliografía históricomédica aunque, probablemente, la visibilidad historiográfica de los trabajos basados en ellas ha sido pequeña hasta bien entrada la última década del siglo XX. Por otra parte, las fuentes no han recibido suficiente autoridad como para desdibujar su asociación con la elaboración de biografías de personajes destacados o con el estudio de grupos o sociedades ágrafas, ni tampoco para desdecir la imposibilidad que tendría la historia oral para formular «grandes hipótesis históricas» ${ }^{3}$.

Los objetivos de la historia basada en fuentes orales son hoy bien diferentes a los que la bibliografía historiográfica les ha asignado durante mucho tiempo. En un plano que podríamos considerar político, la historia oral renace para dar Voz a sectores socialmente silenciados por motivos de clase, raza, lugar de residencia, género, o cualquier otra jerarquía y, por tanto, invisibles en la escritura histórica. Desde un punto de vista teórico, responde al interés por el estudio de la memoria, la representación y la subjetividad. Guarda una directa relación con el trabajo de campo en antropología social y, en general, con las técnicas cualitativas de las ciencias sociales, pero también tiene unas características epistemológicas propias que radican, básicamente, en la importancia que la historia da a elementos tan inasibles en otras ciencias como el tiempo y la memoria ${ }^{4}$.

Durante los últimos diez años, numerosos trabajos, publicados preferentemente como artículos en revistas anglosajonas de historia oral y de historia de la medicina, han hecho aportaciones teóricas y de estudios de casos sobre

2 Una búsqueda en la Bibliografía española de historia de la ciencia y de la técnica http://161.111.141.93/hcien/ (realizada el 26 febrero 2003) arroja un único resultado buscando por el descriptor («historia oral»); la búsqueda por título y cualquier campo («oral, orales, fuente-s oral-es») proporciona otro título. Se trata de RoviRA, B. (1989), Pau Vila: biografia oral, Barcelona, La Campana, 1989 y SuBIELA, C. et al. (1991), "Arqueologia industrial i fonts orals: ¿una compenetració?», en: Arqueologia industrial. Actes del Primer Congrés del País Valencià, Valencia, Diputación de Valencia. En España se edita desde 1989 una revista especializada: Historia, Antropología y Fuentes Orales y existe desde ese mismo año una sociedad científica, el Seminario de fuentes orales (http://www.ucm.es/info/hcontemp/fuentesorales.htm)

3 Prims, G. (1993), «Historia oral». en: Peter Burke (ed.), Formas de hacer historia, Madrid, Alianza editorial, pp. 144-176.

4 LEYDESDORFF, S. (1999), «Gender and the categories of experienced history». Gender and history, 11 (3), 597-611. 
muy diversos aspectos de la salud y la asistencia sanitaria ${ }^{5}$. En una primera aproximación, podríamos decir que han estado más centrados en el estudio de las profesiones que en el de las instituciones y/o los usuarios de los sistemas de salud. En general, las fuentes orales son un recurso fundamental para conocer los estilos de práctica profesional, la forma de actuar frente a situaciones determinadas y para indagar sobre las transgresiones o seguimiento de las normas sanitarias $^{6}$. Entre las publicaciones recientes sobre profesionales sanitarios hay muchas que se centran en grupos que son minoría en sus profesiones, como enfermeros, cirujanas o mujeres profesionales de la medicina ${ }^{7}$. En España, por otra parte, los escasos trabajos basados parcial o totalmente en fuentes orales han tratado sobre la experiencia de médicas y científicas ${ }^{8}$, sobre la relación médico-paciente, o sobre las instituciones y la asistencia médica9 ${ }^{9}$

5 También sobre historia de la ciencia, como MüLLER-HiLl, B. (2001), «Reflections on the use of interviews in the history of science : who will write the history of science», History and philosophy of the life sciences, 23 (1), 105-116 y DOEL, R. E. (2003), "Oral history of American science: A forty-year review», History of Science, 61, 349-378. Una bibliografía de trabajos publicados con anterioridad a 1990: PERKS, R. (1990) Oral history: an annotated bibliography, London, Bristish Library.

6 Ver Leap, N.; Hunter, B. (1993), The midwife's tale. An oral history from handywomen to professional midwife, London, Scarlett Press; ANDERSON, S. (1997), ««I remember it well»: oral history in the history of pharmacy». Social History of Medicine, 10 (2), 331-343; RHODES, M. (2001) «Saber y práctica de la matronería en Gran Bretaña, 1936-1950», en M. Cabré; T. Ortiz (eds.) Sanadoras, matronas y médicas en Europa. Siglos XII-XX, Barcelona, Icaria, pp. 189-216 y los seis primeros capítulos del libro BORNAT, J. et al. (eds.) (2000), Oral history, health and welfare, London, Routledge.

7 Algunos ejemplos sobre enfermeros: MACKINTOSH, C. (1997), «A historical study of men in nursing», Journal of Advanced Nursing, 26 (2), 232-236; ROLPH, S.; J. WALMSLEY; D. ATKINSON. (2002), «'A man's job'?: gender issues and the role of mental welfare officers, 1948-1970», Oral History, 30(1), 28-41; Brown, B.; Nolan, P.; Crawford, P. (2000), «Men in nursing: ambivalence in care, gender and masculinity», International History of Nursing Journal, 5 (3), 4-13. Sobre cirujanas: CASSELL, J. The woman in the surgeon's body. Cambridge-Ma, Harvard University Press, 1998. Sobre médicas: WALKER, L. (2001), «'Conservative pioneers': the formation of the South African Society of Medical Women», Social History of Medicine, 14 (3), 483-505. Sobre matronas: RHODES, M. (2000), «The contribution of professional education and training to becoming a midwife, 1938-1951», en: BORNAT et al. (eds.), pp. 119-138; Leap, Hunter (1993).

8 Magallón Portolés, C. (1998), Pioneras españolas en las ciencias. Las mujeres del Instituto Nacional de Física y Química, Madrid, CSIC; SANTESMASES, M. J. (2000), Científicas en España (1940-1970): profesionalización y modernización social, Madrid, Instituto de la Mujer; OrTiz Gómez, T. et al. (2001), «Ser mujer y médico en la España de los años sesenta», Asparkía, (12), 125-136; Delgado SÁnchez, A.; TÁvora Rivero, A.; Ortiz Gómez, T. 
Un libro que representa muy adecuadamente las líneas de investigación actuales y las perspectivas de futuro, es Oral history, health and welfare, editado por cuatro profesionales de amplia experiencia en el tema, entre ellos Joanna Bornat y Paul Thompson ${ }^{10}$. Su editora y editores recopilan contribuciones sobre médicos, farmacéuticos, especialistas, matronas, enfermeras, pacientes y usuarios del sistema sanitario (casos de sida, invidentes, retrasados mentales o control de natalidad). Los temas de sus capítulos están directamente relacionados con la experiencia de las personas entrevistadas: las razones de sus decisiones, las actitudes, sentimientos o expectativas ante situaciones determinadas, los procesos de búsqueda de asistencia sanitaria y la construcción de sus identidades profesionales. Algunos de ellos se acercan a un tema desde la doble perspectiva de los pacientes y de los profesionales, visión pluridimensional de notable interés historiográfico.

En la introducción al libro, Paul Thompson señala que el uso de las fuentes orales en historia de la medicina permite, al menos, tres cosas importantes: a) construir una historia más compleja, con información de la vida de muy diversas personas (gente común, mujeres, clases medias, minorías, pacientes, cuidadoras y cuidadores, profesionales sanitarios de a pie...) sobre aspectos relacionados con su salud y con los sistemas de atención sanitaria; b) acceder al mundo de las relaciones familiares y personales, a las culturas profesionales, a las formas de relación de los equipos de trabajo, a las formas de práctica y de organización del trabajo sanitario cotidiano; y c) volver sobre áreas ya

(2003), «Las médicas, sus prácticas y el dilema con la feminidad», Estudios de Sociolingüística. Linguas, sociedades e culturas, 4(2), 589-611. Sobre científicas hay publicados bastantes testimonios orales de carácter autobiográfico, como muchos de los artículos del monográfico editado por SANTESMASES, M. J.; FernÁNDEZ VARGAS, V. (2002), Ciencia y tecnología en el CSIC. Una visión de género, Arbor, (679-680), o los trabajos de SALAS, M. (1999), «La mujer y la ciencia: mi propia historia», en: M. Cruz Rodríguez; L. Ruiz Higueras (eds.), Mujer y ciencia, Jaén, Universidad de Jaén, pp. 95-108 y MIQUEO, C. (1999), «Contrastar experiencias: diversidad de modelos para las científicas. Una mesa redonda», en: M. J. Barral et al. (eds.), Interacciones ciencia y género, Barcelona, Icaria, pp. 291-324.

9 Larraz Andía, P. (2002), El Hospital Alfonso Carlos. Pamplona 1936-1939, Tesis de doctorado, Universidad de Navarra; LARRAZ, P.; LEÓN SANZ, P. (2002), «Organización de la asistencia médico-quirúrgica en Navarra a los combatientes en la guerra civil (1936-1939)», en: C. Erro Gasca; I. Mugueta Moreno (eds.), Grupos sociales en la historia de Navarra, relaciones y derechos: Actas del V Congreso de Historia de Navarra, Pamplona, Ediciones Eunate, pp. 377-381; LEÓN SANZ, P. (2000), «La confidencialidad del médico y la información debida a las entidades aseguradoras», Revista de Medicina de la Universidad de Navarra, 44(1), 25-31.

10 BornAt et al. (eds.) (2000). 
trabajadas (instituciones, hospitales, organización sanitaria, organizaciones médicas etc.) para plantear nuevas perspectivas y nuevas preguntas ${ }^{11}$.

La historia basada en fuentes orales supone indagar en la subjetividad, es decir, en un área de actividad simbólica que incluye aspectos cognitivos, culturales y psico-sociales, que no hay que confundir, aunque esté relacionado, con mentalidad, ideología o cultura. Su fuerza radica en la individualidad y la diversidad de la experiencia y en su posibilidad de ofrecer una visión poliédrica de diferentes representaciones culturales, lo cual es muy enriquecedor en la elaboración de relatos prosopográficos. Tiene además una importante consecuencia sobre la historia en general pues, en expresión de Leydesdorff (1999), produce «disonancias» en la historia ya construida, es decir, en el conocimiento histórico sobre un tema. Y estas disonancias son elementos de transformación que favorecen un conocimiento del pasado más complejo al tiempo que ofrecen argumentos críticos sobre lo que constituye la realidad.

Un elemento metodológico de gran importancia cuando se utilizan fuentes orales es tener presente que la historia oral no proporciona datos sino que es expresión y representación de la(s) cultura(s) y contiene no sólo narraciones literales y el recuerdo de hechos concretos, sino también la memoria, la ideología y los deseos inconscientes de las personas que aportan sus testimonios. Así mismo, no hay que olvidar que la mayoría de las fuentes orales son el resultado de una situación interactiva en la que la persona que entrevista y la persona entrevistada confrontan sus propias subjetividades, de tal manera que la experiencia de la investigada siempre está influenciada por la de quien investiga $^{12}$. Por ello, la historia oral supone, casi siempre, ir más allá de la interpretación y significación de las fuentes para tomar parte también del proceso de creación de las mismas.

Un aspecto fundamental de la historia oral radica precisamente en la creación de archivos de fuentes. Los archivos de fuentes orales de interés histórico-médico que existen, en audio y/o video, son de dos tipos: integrados en programas generales de historia oral, o segregados en programas específicamente sanitarios. Son numerosos en EE.UU. y Gran Bretaña, y en buena parte

11 ThOMPSON, P. (2000), «Introduction», en: BorNAT et al. (eds.), pp. 1-20.

12 PASSERINI, L. (1998), «Work ideology and consensus under Italian fascism», en: BoRNAT et al. (eds.) (2000) pp. 53-62; LEYDESDORFF (1999). Sobre subjetividad y fuentes orales ver también BORDERÍAS, C. (1997), «Subjetividad y cambio social en las historias de vida de mujeres: notas sobre el método biográfico», Arenal, 4(2), 177-195 y GAGLIANI, D. (1997), «Mujeres, guerra y resistencia en Italia. Una reflexión historiográfica y una vía de investigación», Arenal, 4(2), 197-222. 
son resultado de programas específicos que, mediante entrevistas en profundidad e historias de vida grabadas en audio, catalogadas y transcritas, recogen la experiencia individual y la memoria de personas, general pero no exclusivamente mayores, sobre cuestiones relacionadas con la salud y el bienestar social13. Por ejemplo, en Gran Bretaña, el Royal College of Physicians tiene, junto con Oxford Brookes University, un archivo con 200 entrevistas de sus colegiados y el Wellcome Trust trabaja en la generación de otro con testimonios de médicos, investigadores y gestores acerca de temas específicos. Existen también archivos generales y temáticos como los programas de fuentes orales sobre SIDA desarrollados en EEUU a partir de 1980. El objetivo es, en todos los casos, generar y conservar las fuentes para su estudio actual y/o futuro y muchos responden a iniciativas de carácter más documental que historiográfico. Aunque la tendencia actual más frecuente por parte de profesionales de la historia es crear sus propias fuentes en función del tema de estudio y las preguntas de partida, la posibilidad de utilizar este tipo de archivos puede ser de mucho interés ${ }^{14}$. En cualquier caso, procurar la conservación de las fuentes generadas de uno $\mathrm{u}$ otro modo $\mathrm{y}$, a ser posible, organizar un archivo específico, podría convertirse también en un proyecto para la historia de la medicina española.

2. LAS FUENTES ORALES PARA EL ESTUDIO DE LAS MUJERES PROFESIONALES DE LA SALUD

La asociación entre biografías colectivas, historia oral e historia de las mujeres en la medicina y en las actividades sanitarias, tiene una coherencia que

13 Sobre archivos de fuentes orales: THOMPSON, P. (1991), «Oral history and the history of medicine: a review», Social history of medicine, 4(2), 371-383; THOMPSON (2000); MckenzIE, D.; PIfAlO, V. (1998), «The Oral History Program: I. Personal views of health sciences librarianship and the Medical Library Association», Bulletin of the Medical Library Association, 86 (2), 166-82; HANNAWAY, C. (1996), «The NIH AIDS Oral History Project: documenting the NIH response to AIDS (1981-1991)», Watermark, 19 (4), 118-21; RICKARD, W. (2000), «HIV and Aids testimonies in the 1990s», en: BORNAT et al. (eds.), pp. 227-248; TANSEY, T. et al. (1997), "What is a witness seminar?», en: Wellcome witnesses to twentieth century medicine, London, Wellcome Trust, vol. 1, p. i-v, en The guide to history of medicine resources on the Internet $<\mathrm{http}: / /$ medhist.ac.uk/ $>$ (consultado en junio 2003) hay enlaces con 18 programas británicos y norteamericanos relacionados con salud, enfermedad y medicina. Sobre archivos de fuentes orales en historia de la ciencia: DOEL (2003), p. 351-361.

14 Un ejemplo interesante es el trabajo de WATKINS, E. S. (1998), On the pill: a social history of oral contraceptives, 1950-1970, Baltimore, Johns Hopkins University Press, que utiliza entrevistas de un archivo de planificación familiar, además de las suyas propias. 
viene dada por la relativa frecuencia con la que han estado asociadas. Probablemente el primer trabajo sobre la actividad profesional de las médicas en el siglo XX, escrito con una perspectiva histórico-social y sobre la base de entrevistas en profundidad, sea el libro que editaron en 1982 tres investigadoras norteamericanas con el expresivo título de In her own words: oral histories of women physicians ${ }^{15}$. En él se recogen los relatos de nueve médicas de tres generaciones distintas acerca de su vida y su práctica profesional. Estas historias de vida están escritas en primera persona y se complementan con sendos capítulos de discusión que las analizan de forma cruzada y las sitúan en el contexto profesional y social de su tiempo. Entre las editoras se encuentra la profesora norteamericana Regina Morantz-Sanchez, una de las precursoras de la institucionalización de la historia de las mujeres en Estados Unidos y activa participante en los primeros debates internacionales sobre la relación entre historia, mujeres y feminismo ${ }^{16}$.

Como es bien sabido, a lo largo del siglo XX la medicina se ha convertido en occidente en una profesión progresivamente feminizada. El proceso ha seguido, en general, dos fases: una de crecimiento muy lento, con momentos incluso de estancamiento, que se prolonga con variaciones hasta la década de 1960 y otra consecutiva de crecimiento exponencial que coincide, en Europa, con la expansión numérica de la profesión dentro del contexto de los sistemas de asistencia sanitaria pública. A lo largo de los últimos diez años numerosas investigaciones se han centrado en analizar esta segunda fase atendiendo a aspectos nuevos en los que los análisis sociológicos, antropológicos e históricos se han integrado o bien han ofrecido perspectivas complementarias. Las publicaciones se insertan en una tendencia de investigación interdisciplinar sobre el trabajo de las mujeres en general y sobre el trabajo de las mujeres en

15 Morantz-Sanchez, R. M.; Pomerleau, C.; Fenichel, C. H. (1982). In her own words: oral histories of women physicians. Westport, Conn: Greenwood Press.

16 Por ejemplo MoRANTZ-SANCHEZ, R. (1974a), «The perils of feminist history», Journal of interdisciplinary history, 4(4), 649-660. Participó en el primer congreso de de historia de las mujeres de Berkshire y su intervención fue publicada como capítulo de uno de los libros más influyentes para la historia de las mujeres y la salud de los años 70: MORANTZ, R. (1974b), «The lady and her physician». en: M. HARTMAN; L. BANNER (eds.), Clio's consciousness raised. New perspectives on the history of women, New York, Harper \& Row, pp. 38-53. Sobre historia de la historiografía de la medicina y género trato en ORTIZ GómEZ, T. (2004), «Historia de la medicina e historia de las mujeres», en: M. I. del Val Valdivieso et al. (coords.), La historia de las mujeres, una revisión historiográfica, Valladolid, Universidad de Valladolid, pp. 105-120, p. 112-113. 
las profesiones de base universitaria (científicas de diversas disciplinas y profesoras de distintos niveles) en particular.

Al menos cuatro libros, publicados recientemente, ejemplifican estos diferentes y productivos modelos disciplinares de acercamiento. ${ }^{17}$ Las preguntas que subyacen a todos ellos, y a muchos otros trabajos sobre el mismo tema publicados en los últimos años, son básicamente dos: por una parte, si la entrada masiva de mujeres ha transformado la práctica y la estructura de la medicina y, por otra, y menos ambiciosamente, si las médicas practican la profesión de modos diferentes a los médicos. Otra pregunta indaga las formas de vinculación de la vida privada o doméstica y la vida profesional, la relación entre lo familiar y lo laboral ${ }^{18}$. Todos los trabajos aplican a su análisis una perspectiva de género.

La hipótesis, contrastada sobradamente en todas las investigaciones, es que existen modelos distintos de entender la profesión entre hombres y mujeres, y que, a su vez, las posturas femeninas no son idénticas sino diversas entre si en cada contexto estudiado. Esta constatación hace que la respuesta a la primera pregunta se torne más compleja y que exija, también su reformulación en términos menos globales. En cualquier caso, y en general, la asociación entre medicina y autoridad masculina sigue siendo una norma a finales del siglo XX, más allá de la entrada masiva de mujeres.

Joan Cassell, una antropóloga de Washington University, lleva a cabo un estudio sobre 33 cirujanas norteamericanas de diferentes especialidades y edades que ejercían a principios de los años 90, utilizando como método la observación de cada una de ellas durante varias jornadas de trabajo y la realización de entrevistas complementarias ${ }^{19}$. El objetivo es estudiar las diferencias y similitudes entre la práctica y las identidades de mujeres y varones especialistas así como entre la práctica de las propias cirujanas en uno de los espacios profesionales más masculinizados de la medicina estadounidense.

La historiadora Ellen More publicó en 1999 su libro sobre las médicas norteamericanas a lo largo de siglo y medio, tomando como ejemplo, precisamente contextualizado, el condado neoyorkino de Rochester. Los elementos

17 CASSEll (1998); More (1999), Restoring the balance. Women physicians and the profession of medicine, 1850-1995, Cambridge-MA, Harvard University Press; RISKA, E. (2001a), Medical careers and feminist agendas. American, Scandinavian and Russian women physicians, New York, Aldine de Gruyter; PRINGLE, R. (1998), Sex and medicine: gender, power and authority in the medical profession, Cambridge, Cambridge University Press.

18 CASSELL (1998) y MORE (1999).

19 CASSEll (1998). 
que combina son muchos y se detiene en analizar las formas de segregación y las identidades construidas según género y raza, así como su transformación en el periodo estudiado, para concluir que la vida de las médicas en este largo periodo representa, en su diversidad, una constante búsqueda de equilibrio entre lo personal, lo colectivo y sus intereses profesionales.

El libro de Elianne Riska, una socióloga finlandesa experta y muy productiva en el tema, es un estudio comparado de las médicas en seis países que han tenido procesos de feminización muy diferentes debido a su diferente estructura socioeconómica y sanitaria ${ }^{20}$. Se centra en el análisis de los últimos 40 años sobre la base de datos estadísticos para el conjunto de la profesión y las especialidades. Tiene también un capítulo donde, a través de entrevistas a médicas finlandesas especialistas en Atención Primaria, desvela el papel del género en las formas de organización científica de la especialidad y los procesos de segregación de las mujeres. En una línea similar, pero basado exclusivamente en fuentes orales, sobre todo entrevistas semi-estructuradas a 150 médicas australianas y británicas a comienzos de los años 90, se encuentra el interesante libro de la socióloga Rosemary Pringle ${ }^{21}$. En él se estudia la vida profesional de especialistas en medicina de familia, psiquiatría, anestesia y cirugía, y los efectos producidos por el sistema de género en sus trayectorias. Señala la autora la universalidad de las experiencias de las médicas en su intento de buscar un equilibrio entre lo familiar y lo profesional, sus dudas ante su propia promoción, y la voluntad y la dificultad de practicar una medicina que muchas de ellas denominan como más humana.

Como señala Pringle, la mayoría de los trabajos basados en fuentes orales no buscan verdades absolutas acerca de las diferencias profesionales entre varones y mujeres, sino que pretenden indagar en la producción discursiva de la diferencia y en las subjetividades e identidades que se construyen en relación con estos significados ${ }^{22}$, entendiendo que ello contribuye al mejor conocimiento de la profesión y de la asistencia sanitaria. Los trabajos que responden a una metodología histórica buscan, además, establecer cronologías y situar los hechos estudiados en su contexto, señalando los procesos de cambio y transformación de las prácticas a lo largo del tiempo. En este sentido, el

20 RISKA (2001a). Ver también RISKA, E. (2001b), «Towards gender balance: but will women physicians have an impact on medicine?», Social Science and Medicine, 52(2), 179187. Un trabajo colectivo anterior es RISKA, E.; WEGAR, K. (eds.) (1993), Gender, work and medicine. Women and the medical division of labour, London, Sage.

21 PRINGLE (1998).

22 PRINGLE (1998), p. 221. 
libro de Ellen More es modélico, como lo son también algunos artículos recientes sobre médicas sudafricanas y británicas ${ }^{23}$.

La mayoría de estos trabajos plantean su investigación desde puntos de vista constructivistas, ponen el énfasis en las relaciones de género que estructuran la profesión y subrayan la elaboración de discursos cuyo objeto es definir las profesiones en términos de masculinidad o feminidad. De este modo, es factible centrarse en el estatus de las mujeres en las profesiones sanitarias y desvelar las prácticas sociales y culturales que definen su papel como mujeres dentro de ellas. En el campo de la historia, esta perspectiva tiene una gran importancia y complementa otros acercamientos frecuentes, como los de tipo neo-weberiano o los basados en la teoría de la socialización que se han utilizado para explicar, respectivamente, los mecanismos de clausura (o exclusión) y las diferencias de género dentro de las profesiones ${ }^{24}$.

En España, no existen apenas trabajos de carácter histórico sobre médicas del siglo XX excepto las biografías recientes sobre la médica y anarquista Amparo Poch y Gascón o los míos propios ${ }^{25}$. Acerca de las mujeres científicas, dos libros cubren, de manera complementaria, gran parte del siglo XX desde principios de siglo hasta los años setenta ${ }^{26}$. Ambos, pero especialmente el de Santesmases dedicado a los años del franquismo, utilizan entre sus fuentes entrevistas realizadas a mujeres investigadoras en diversas ramas de las ciencias naturales y experimentales.

23 Walker (2001); ElStON, M. A. (2001), «"Run by women, (mainly) for women”: medical women's hospitals in Britain, 1866-1948», Clio Medica, 61, 73-107.

24 Sobre los marcos de análisis en sociología de las profesiones: RISKA (2001b).

25 Rodrigo, A. (2002), Una mujer libre: Amparo Poch y Gascón, médica anarquista, Barcelona, Flor del viento. Entre mis trabajos más recientes: ORTIZ GóMEZ et al. (2001); Delgado SÁnchez; TÁvora Rivero; Ortiz Gómez (2003). En una línea divulgativa: De JuAn CAstrillo, A. (1998), La colegiación femenina. Las primeras mujeres del Colegio Oficial de Médicos de Palencia, Palencia, Ilustre Colegio Oficial de Médicos; LóPEZ DE LETONA, C. (1999), Trinidad Arroyo Villaverde y la ofatalmología española, San Sebastián, Instituto Universitario de Oftalmología Aplicada.

26 MAgAllón (1998) y SANTESMASES (2000). También utilizan fuentes orales para estudiar a maestras y profesoras SAN ROMÁN GAGO, S. (2001), La maestra en el proceso de cambio social de transición democrática: espacios histórico generacionales, Madrid, Instituto de la Mujer-Ministerio de Asuntos Sociales y GARCÍA DE LEÓN, M. A.; GARCía DE CoRTÁzAR, M. (2001), Las académicas. Profesorado universitario y género, Madrid, Instituto de la Mujer-Ministerio de Asuntos Sociales. 
3. LAS MÉDICAS ESPAÑOLAS EN LA SEGUNDA MITAD DEL SIGLO XX: IDENTIDADES E INTEGRACIÓN PROFESIONAL

En mi reciente trabajo de investigación sobre las médicas españolas del siglo veinte, realizado en parte dentro de un equipo interdisciplinar y centrado en la segunda mitad del siglo $\mathrm{XX}^{27}$, están presentes muchos de los planteamientos de las autoras y trabajos comentados anteriormente, aunque su lectura fuera en casi todos los casos posterior al inicio de la investigación.

Nuestro objetivo era estudiar la actividad profesional de las mujeres que ejercen y han ejercido la medicina en los últimos 40 años del siglo $\mathrm{XX}$, recabar y analizar su experiencia personal, comparar la situación de finales de los sesenta y la de comienzos de los noventa, e insertar todo ello dentro de un contexto historiográfico que ha sido, a su vez, objeto de estudio ${ }^{28}$.

Las fuentes orales formaron parte de un repertorio heurístico más amplio en el que incluimos fuentes estadísticas, archivísticas, bibliográficas y hemerográficas ${ }^{29}$, y fueron creadas por el equipo investigador utilizando dos técnicas diferentes: entrevistas en profundidad (16 entrevistas a médicas de la generación de los sesenta) y grupos operativos de entre 6 y 8 integrantes (dos con médicas mayores, uno en Valencia y otro en Madrid, y cinco más con especialistas jóvenes de Madrid y Granada). Las entrevistas, que realicé personalmente, nos permitieron construir historias de vida y trayectorias profesionales individuales de una serie de médicas que empezaron a ejercer a finales de los años sesenta ${ }^{30}$. Por su parte, los grupos operativos (realizados por

27 La investigación se llevó a cabo entre 1998 y 2003 gracias a un proyecto del Programa Sectorial de Estudios de las Mujeres y Género del Ministerio de Trabajo y Asuntos Sociales (RS/EL exp: 51/97) y a sendas ayudas del Plan Andaluz de Investigación y Plan Propio de la Universidad de Granada en el año 2002.

28 Algunos resultados de esta investigación son: ORTIZ GómEZ et al. (2001); Delgado SÁnchez; TÁvora Rivero; Ortiz Gómez (2003); Ortiz Gómez, T. (2004); OrTiz Gómez, T. (2001a), «Espacios de sociabilidad femenina en la profesión médica en los siglos XIX y $\mathrm{XX}$ », en: V Frías (ed.) Las mujeres y la ciencia ante el siglo XXI, Madrid, Universidad Complutense, p. 71-90.

29 El detalle de las fuentes y la metodología en: OrTIZ GóMEZ, T. et al. (2000), Trabajo, género y medicina. Actividad profesional de las médicas españolas en la segunda mitad del siglo $X X$. Memoria de investigación inédita, Instituto de la Mujer. La memoria se puede consultar en el centro de documentación del Instituto de la Mujer-Ministerio de Asuntos Sociales.

30 Las entrevistadas fueron elegidas entre las mujeres que figuran inscritas entre $1950 \mathrm{y}$ 1970 en los libros de registro de los Colegios de Médicos de Madrid, Barcelona y Valencia. 12 de ellas fueron elegidas al azar y cuatro por su pertenencia a la AEMM. Realicé las entrevistas entre octubre de 1999 y mayo de 2000. Mi aprendizaje del uso de fuentes orales en sus 
las expertas del equipo, las doctoras Ana Távora y Ana Delgado) nos acercaron a la creación de discursos colectivos de médicas con rasgos profesionales comunes, como la especialidad, la experiencia asociativa o la pertenencia generacional $^{31}$.

La identidad, pieza importante de nuestro análisis teórico, la entendemos como un proceso constante de interpretación y apropiación simbólica de la realidad, como una forma de autorrepresentación consciente, así como el resultado de un proceso de interacción social entre las trayectorias individuales y los sistemas de organización institucional. Reconocer la afirmación y conformación de las identidades individuales y colectivas es de gran valor para la comprensión de la sociedad en su conjunto y de las actividades laborales ${ }^{32}$. Las profesiones suelen tener identidades sexuadas, (o más raramente ambiguas) pero nunca neutras y adoptan valores que responden a códigos sociales masculinos o femeninos. Algunas aportaciones han demostrado que la medicina ha construido una identidad claramente masculina y consideramos pertinente estudiar qué tipo de identidades habían construido y estaban construyendo las mujeres dentro de esta profesión ${ }^{33}$.

Para analizar la construcción de identidades individuales o colectivas hemos tenido presente también, como los trabajos citados en el apartado anterior, la diferencia entre las mujeres, un dato que, como ha señalado Joan Scott recientemente, se ha convertido en una categoría analítica para el feminismo, pues «al describir diferencias entre las mujeres se establece el hecho de las identidades separadas, pero también se establece el carácter relacional de la diferencia ${ }^{34}$. También hemos tenido presente que la identidad no es inherente, por ejemplo, al cuerpo, a la nacionalidad o a la actividad profesional, sino que se construye, discursiva e históricamente, por contraste. Parafraseando a

aspectos teóricos, técnicos y, también, emocionales, me decidió a posponer el análisis de las entrevistas y dejar transcurrir un tiempo después de su realización.

31 Sobre la metodología de grupos utilizada ver también Delgado SÁNCHEZ; TÁvorA RIVERO; ORTIZ GÓMEZ (2003), pp. 594-596.

32 Ortiz Gómez, T. (2001b), «El género, organizador de las profesiones sanitarias», en: C. Miqueo et al (eds.), Perspectivas de género en salud, Madrid, Minerva, pp. 53-75, (p. 5658). LlonA, M. (2002), Entre señorita y garçonne historia oral de las mujeres bilbaínas de clase media, (1919-1939), Málaga, Universidad de Málaga.

33 Remito a la bibliografía citada en ORTIZ GómEZ (2001b), pp. 56-58.

34 Scott, J. W. (1996), Feminism and history, Oxford, Oxford University Press, pp. 8-9. Existe una versión española del capítulo introductorio de este libro: SCOTT, J. (1997), «Feminismo e historia», Anuario de Hojas Warmi (8), 109-122. 
$\mathrm{Scott}^{35}$, podríamos decir que ser médica significa, probablemente y entre otras cosas, no ser enfermera ni matrona $y$, en general, significa también no ser médico (varón). Una idea que liga género e identidad en el diseño de nuestra investigación, es el hecho de que dentro de la profesión médica han operado, y operan, los mismos sistemas de género que en la sociedad en su conjunto, es decir, que dentro de la profesión médica «la identidad de género se crea y se recrea [...]; los individuos no acceden a la profesión con una identidad fija y firmemente arraigada» sino que esta se redefine en el ámbito laboral ${ }^{36}$.

Las preguntas sobre si las médicas construyen identidades colectivas diferenciadas de las masculinas, qué identidades individuales construyen y qué tienen en común y de diferentes, han sido en este trabajo una de las claves de la investigación. Prestamos especial atención a los espacios intraprofesionales marcados por el género, concretamente, la asociación de médicas que existió en los años sesenta y setenta y una serie de especialidades caracterizadas por su diferente distribución de especialistas según sexo: muy feminizadas, como pediatría y psiquiatría, o masculinizadas, como cirugía y gestión sanitaria, o con similar participación de varones y mujeres y con ideologías de trabajo más ambiguas desde un punto de vista de género, como la especialidad medicina de familia.

Parte de las preguntas de partida giraban en torno a la comparación y las conexiones identitarias y genealógicas entre dos generaciones de mujeres profesionales. Buscábamos elementos de continuidad y cambio entre las médicas, partiendo de la hipótesis de que, a pesar de los 30 años de distancia, algunos aspectos de su vida profesional se mantenían, al tiempo que habría otros absolutamente nuevos. Nos preguntábamos acerca de la existencia de un reconocimiento de autoridad entre médicas de diferentes generaciones y si las médicas de mayor edad y experiencia fueron para las más jóvenes modelos de práctica profesional ${ }^{37}$. También indagamos sobre las formas de conciliación de la vida profesional y privada (su opción de vida en pareja o no, con o sin hijos). Y en qué medida todos estos aspectos habían interactuado en sus trayectorias profesionales y vitales.

Voy a comentar sucintamente algunos resultados referidos a la Asociación Española de Mujeres Médicos (AEMM) y a la práctica de las mujeres que

35 SCOTT (1997), p. 116, lo ejemplifica diciendo desde su perspectiva estadounidense que «ser blanca significa no ser negra».

36 McDowell, L. (1999), Género, identidad y lugar, Madrid, Cátedra, p. 199.

37 Este tema resultó bastante inusual en la bibliografía revisada, tan solo tratado por MoRANTZ-SANCHEZ (1982). Sobre nuestros resultados: ORTIZ et al. (2001). 
ejercían en los años sesenta, prestando atención a los aspectos más significativos que introduce el uso de fuentes orales.

Las asociaciones de médicas nacieron como un espacio de segregación y/o de exclusión y se transformaron, en unos países y momentos más que en otros, en espacios de libertad profesional y personal. En algunos países han sido, también, espacios de poder profesional ${ }^{38}$. La Asociación Española de Mujeres Médicos (1965-1978), la segunda de su tipo que existió en el país tras la Asociación de Médicas Españolas (1928-1936), fue uno de los primeros grupos independientes de mujeres en la España franquista. Se constituyó oficialmente en Valencia con los objetivos de facilitar la cooperación entre las médicas de España y del mundo, y de estudiar los «problemas que interesan especialmente a las mujeres médicos relacionados con la mujer, el niño y la medicina social ${ }^{39}$. Algunos aspectos significativos de su trayectoria son los siguientes ${ }^{40}$ :

Durante su existencia tuvo 160 socias de toda España, una cifra que a finales de los sesenta suponía algo más del $10 \%$ de las profesionales del país. Editaron una revista (Actividades de la Asociación Española de Mujeres Médicos, 1965-1971), organizaron dos congresos nacionales en 1971 y 1975 y realizaron numerosas actividades de carácter divulgativo. La AEMM formó parte de la Medical Women's International Association (1919-) a cuyos congresos asistieron representaciones de socias de forma habitual. A través de su revista y sus actividades construyeron genealogías de práctica médica femenina, pero no se relacionaron con otros grupos españoles de mujeres que existían en su tiempo, ni siquiera los de carácter independiente, como la Asociación de Mujeres Universitarias, legalizada de nuevo tras la guerra en 1953, o el Seminario de Estudios Sociológicos, fundado en 1960, con los que compartían unas raíces comunes ${ }^{41}$.

38 Como en el caso estadounidense: More, E. S. (1990), «The American Medical Women's Association and the role of the woman physician, 1915-1990», JAMWA, 45 (5), 165-180.

39 AEMM (1967), Estatutos, Valencia, s.e., p. 3.

40 Remito a mis trabajos: OrTIZ GóMEZ (2001a, 2001b); OrTIZ GóMEZ et al. (2000 y 2001).

41 Sobre estas asociaciones y sobre los vínculos dentro del movimiento de mujeres en España: MORENO SARDÁ, A. (1988), «La réplica de las mujeres al franquismo» y PARDO, R. (1988), «El feminismo en España: Breve resumen», ambos en: P. Folguera (ed.), El feminismo en España: dos siglos de historia, Madrid, Pablo Iglesias, pp. 85-110 y 133-141 respectivamente; FAGOAGA, C. (1985), La voz y el voto de las mujeres, Barcelona, Icaria, pp. 143-192; MAILlaRD, M. L. (1990), Asociación Española de Mujeres Universitarias (1920-1990), Madrid, AEMU/Instituto de la Mujer, pp. 11-32; OrTIZ GómEZ, T. (1988), «Asociacionismo médico femenino en España: La Asociación de Médicas Españolas (1928-36) y su fundadora, 
Su actividad descartaba, a diferencia de la mayoría de sociedades profesionales, la reivindicación de cuestiones laborales. A pesar de ello, llevaron a cabo una afirmación continua del importante papel de las mujeres en la profesión y redefinieron y dieron valor a la diferencia de ser mujer y a los significados sociales que tenía en su tiempo. Las médicas de la AEMM asumieron las diferencias convencionales entre los sexos, entendiendo que eran de orden social y natural, pero no restrictivas, sino positivas y adecuadas para practicar la medicina.

En el grupo de discusión realizado en 1999 con 13 antiguas socias, de edades comprendidas entre 68 y 80 años, todas jubiladas, las médicas calificaban su experiencia asociativa como una iniciativa de carácter médico y cultural en la que «lo femenino» (refiriéndose a lo personal y familiar) no tenía espacio. Una actividad que realizaron sin tensiones internas ni externas, que no colisionó con su vida familiar y que realizaron de buen grado gracias al continuo empuje de su fundadora, la pediatra valenciana Lola Vilar (1900-1990). El discurso grupal pone de manifiesto su conciencia de las dificultades que tenían por el hecho de ser mujeres, aunque las sitúan en el mundo doméstico y familiar más que en el profesional. La estrategia para superarlas se basaba en evitar los enfrentamientos, asumiendo su rol (en tanto que mujeres) e intentado transformar las condiciones cuando se presentaba la ocasión: «era una resistencia pasiva, no declarada, porque declarada no la podías hacer, pero bajo mano, sí» ${ }^{42}$.

Del discurso grupal de las que fueron socias y, más todavía, de los documentos de archivo e impresos de la AEMM, emana un relato homogéneo y un tanto idílico respecto a la vida asociativa, que se representa sin fisuras. Por su parte, los relatos personales generados en las entrevistas en profundidad, permiten matizar este discurso haciendo emerger los procesos de negociación, la disparidad y el conflicto entre ellas, las razones de la adscripción individual o las dificultades profesionales. Todo ello abunda en una imagen de la asociación y de la práctica médica de las profesionales de la época más compleja y plural.

Las razones de la afiliación y la diferente vinculación de las socias hacen ver una diversidad bastante ajena a cualquier hipótesis generalizadora sobre una conciencia colectiva o un compromiso con respecto a los fines de la asociación, y amplían el significado de la participación en los primeros años de

Elisa Soriano (1891-1964)». en: Actas del VIII Congreso Nacional de Historia de la Medicina. Murcia, diciembre de 1986, Murcia, s.e., vol. I, pp. 595-604. OrTIZ GóMEZ, T. (1999), «Elisa Soriano Fischer, una médica feminista», Mujeres y Salud, 5 (febrero), 16-17.

42 Grupo operativo de antiguas socias de AEMM, (mayo de 1999). Ortiz et al. (2001). 
la sociedad. Al mismo tiempo, muestran las redes de contactos que movieron las fundadoras para llevar adelante su iniciativa.

«Yo me hice porque doña Lola Vilar le daría la lata a mi marido [también médico], «que venga tu mujer, que venga tu mujer», entonces me hice yo de la asociación» ${ }^{43}$

«... porque como las mujeres siempre parece que estábamos un poco..., pues por defendernos un poco, querían ellas, porque yo no, porque la realidad es que yo me he llevado maravillosamente siempre bien con mis compañeros, realmente para mí no hacía falta crear aquello, pero me lo pidieron y yo ... pues éramos mujeres y eran amigas, pues adelante» ${ }^{44}$.

«Ah!, sí, entonces surge Lola Vilar y dice que están haciendo una asociación de mujeres médicos y yo dije «mira, vomito solo de decir asociación de mujeres», lo siento, un momento, es un nombre que no le daría nunca, entonces cogí los reglamentos (...) y cogí tal, y la primera asociación mundial que hubo de mujeres médicos en la ultima guerra europea tuvo un papel como tienen ahora las ONG, eso me convenció. Ese papel de apoyo, de quitar entuertos que se hacen socialmente, ese aspecto social tan fuerte que tenía... $\rangle^{45}$.

Un elemento que ayuda a explicar el escaso impacto que tuvo la AEMM entre generaciones posteriores de médicas, así como el hecho de que desapareciera al mismo tiempo que la democracia y un nuevo movimiento de mujeres se organizaban en el país, es la idea de algunas de sus socias de que ya en su momento los planteamientos de la asociación, ya fuera por defecto o por exceso, resultaban un tanto extemporáneos. Para algunas, el carácter poco reivindicativo, era un inconveniente. Una de las socias más jóvenes, licenciada en 1963, relata su desencanto ante lo que le pareció una organización poco activa, que no difundía bien la información y que no luchaba por mejorar la situación de las mujeres profesionales, una aspiración que también parece ineludible en otros testimonios:

«...para mi fue una decepción porque yo creí, yo tenía más esperanzas de que eso funcionase de alguna forma, no para que me beneficiase en nada, pero sí como un dato de solidaridad» ${ }^{46}$.

«yo creo que la mujer [...] para poder hacerse oír está bien que fuera en una asociación de mujeres, pero mientras que la mujer no se hace oír, ni como mujer ni

43 Entrevista CP, licenciada en 1954, (mayo 2000).

44 Entrevista EA, fundadora, licenciada en 1950, (octubre 1999).

45 Entrevista JP, socia, licenciada en 1946, (octubre de 1999).

46 Entrevista OF, socia, licenciada en 1963, (octubre de 1999). 
como médico, ni como nada ..., para comenzar estaba bien que comenzara a hacer ruido y después que le permitieran insertarse en todas las sociedades. Tu mira el fichaje de los señores de todos los colegios de Médicos, no hay una sola mujer que tenga un cargo de relevancia en ningún, este, en ninguna sociedad médica. En ninguna academia médica. Quizás haya una golondrina, ¿no?, que no hace verano dicen, ¿no?» ${ }^{47}$.

La dificultad de compartir entre mujeres y, más aún, disentir entre ellas en el espacio profesional/público, está también presente en el discurso y se manifiesta en forma de recelos y, quizá también, de temor a la segregación

«Hubo un par... que chillaron y no, aquello no es de grato recuerdo, seguramente por eso lo tengo en el olvido. Doña Lola Vilar era una señora que no se merecía eso, porque era una señora maternal, dulce, aunque tenía un aspecto ..., era muy dulce y muy maternal, pero hubo unas jóvenes valores, una histérica, empezaron a chillar allí y ya, yo dejé de ir, no me resultó grato el ambiente y pensé «tiene razón Amparo, con los hombres se está mejor», porque con los hombres no sacamos los trapos sucios de mujeres $\rangle^{48}$.

Las entrevistas permiten ver, por otra parte, las dificultades, las renuncias y las formas de discriminación experimentadas por las médicas en el ámbito profesional, apenas esbozadas en el discurso grupal. Vislumbramos también los confusos límites entre lo profesional y lo familiar, ámbito éste donde las médicas de esta generación sitúan los principales obstáculos a su promoción y desarrollo laboral. La mayoría de las entrevistadas y participantes en los grupos de discusión estaban casadas cuando ejercían su profesión y, como era común entre las profesionales de su tiempo ${ }^{49}$, sus maridos eran en su mayoría colegas. Una de las médicas de más edad, socia de AEMM, que estuvo en activo hasta finales de los años sesenta, tras licenciarse en 1931 y formarse como pediatra la Escuela Nacional de Puericultura en 1932, ejerció en realidad durante muy poco tiempo esta especialidad, ya que después de su matrimonio comenzó a trabajar en el laboratorio que su marido montó en el domicilio familiar

«[...] mi marido me dijo un día «tengo que tomar un ayudante, pero si es médico me va a costar un dinero», digo «pues no te preocupes porque te voy a ayudar y no te voy a cobrar» $[\ldots]$. Pero es que a mi no me gusta el laboratorio. Quería que

47 Entrevista LM, socia, licenciada 1943, (octubre de 1999).

48 Entrevista CP.

49 GARCÍA DE LEÓN (2001), RisKA (1993). 
fuera yo, porque así estoy más tiempo en casa y puedo vigilar a los hijos y, efectivamente, pues trabajé en el laboratorio y, además, ¡ cómo se trabajaba!» ${ }^{50}$.

Una experiencia similar, con matices distintos, fue puesta de manifiesto por una participante en uno de los grupos de discusión:

«yo hice análisis [en el laboratorio anejo al domicilio familiar] hasta que mi marido falleció, entonces me dediqué a la pediatría, que era la especialidad que a mi me gustaba» ${ }^{51}$.

El papel de «ayudante» de los maridos médicos, además del de madre y de cuidadora de los hijos, se repite en varias entrevistas ${ }^{52}$. Si lo segundo se acepta, en general, con lo que podríamos denominar naturalidad, ser ayudante médico generaba frecuentemente conflictos y renuncias profesionales para las mujeres:

«Hubo un congreso en Estados Unidos y México, yo lo veía que no podía más, que él [marido y médico de la misma especialidad] tenía que irse a Estado Unidos porque aquello era, había sido para él la gloria, vamos, y yo dije: « bueno, vete tú», yo tenía ya tres niños, «y yo me quedaré a cargo de todo, de la clínica de casa y de hacer los seguros y de las cosas, pero no te preocupes que yo me quedaré aquíı»». «[...] Yo acostumbraba a manejarme allí yo sola [en mi consulta], con mis enfermos y con mis operados y él me quitaba las cosas de las manos, iporque él las hacía mejor! (risas), ! Bueno!, no tuve valor para decirle: " siéntate y déjame tranquila», y siempre tenía que ir de ayudante (...) Y venga a protestar y venga a criticar $[\ldots]$ y al final, cuando se presentó una plaza sin cirugía de un compañero la cogí, porque esto no sirve más que para disgustos y para tensiones y para pasármelo mal» $[\ldots]\rangle^{53}$

«(...) continué ayudando a mi marido y continué yo ayudando hasta, o sea, estuve unos años sin hacer nada, claro, le ayudaba a él (...) Bueno, siempre que él operaba fuera, particularmente, pues le ayudaba. (...)A mi me sabe mal porque, en realidad, yo de mi especialidad he hecho muy poco (...) quiero decir que ya llevo

50 Entrevista BL, licenciada en 1931, (octubre 1999).

51 Grupo operativo de antiguas socias de AEMM.

52 Otro aspecto de la estrecha relación entre lo familiar y lo profesional es el papel de las esposas, no profesionales, como ayudantes en las consultas de sus maridos médicos, tanto en el siglo XX como en siglos anteriores, tal y como señalan ELSTON, M. A. (1983), The wives of fortunate men, Xerocopy, Thesis (M.A.)University of Essex, y SIENA, K. P. (2001), "The "foul disease" and privacy: The effects of venereal disease and patient demand on the medical market place in early modern London», Bulletin of the History of Medicine, 75, 199-224.

53 Entrevista DL, licenciada 1955, (mayo 2000). 
unos años pues que en fin, que yo he estado aparcada, aparcada, porque sí, porque yo a mi marido pues le he ayudado siempre, pero.... ${ }^{54}$

Trabajar como ayudante ni era exclusivo de la relación entre esposos médicos, ni era exclusivo de las mujeres, sino que respondía a la forma vigente de jerarquización de la profesión y a las múltiples y sinuosas vías de aprendizaje de las especialidades que estuvo en vigor hasta finales de los años setenta. Pero sí que muestra, con claridad, el doble sistema jerárquico, de rango y de género, al que estaban sometidas las mujeres. Ninguna mujer de las entrevistadas hace referencia a una relación con su esposo médico (casi siempre compañeros de carrera) en la que figure él como ayudante. Tampoco como ayudante en las tareas domésticas, que las mujeres resolvían por su cuenta mediante ayuda asalariada y/o redes familiares. La situación no era, en cualquier caso, exclusiva de la profesión médica pues, como ha señalado la antropóloga Teresa del Valle, los varones españoles de más de 65 años nunca vieron comprometida su carrera por la asunción de tareas familiares, ni registraron obstáculos de este tipo en el desarrollo de sus trayectorias laborales ${ }^{55}$.

Quizá por todo ello, las médicas construyen en sus relatos orales un modelo ideal de excelencia profesional ligado a la soltería, a pesar de reconocer algunos inconvenientes asociados en su tiempo, tanto a ser soltera como, también, a ser buena profesional. Este modelo de excelencia, que en su mayoría lamentan no haber alcanzado, se define con elementos que van más allá del trabajo clínico que desarrollaron e incluye el estudio continuado y sistemático, la actividad investigadora, la difusión científica y la participación en congresos ${ }^{56}$ :

«... era muy lista, aprendió [en el extranjero] yo la oía conferencias, daba unas conferencias, unas diapositivas graciosísimas, tenía esa cosa de venir de América, de saber muy bien el inglés y en España, en aquel momento éramos unos papanatas, no sabíamos nada y, claro, pues esa cosa suficiente..., yo creo que ella lo aguantó porque era muy, muy valiente y nada parecido a la media, y un esfuerzo brutal, pero ella lo hizo. Era soltera, y todas sus energías las invertía en reivindicar

54 Entrevista FB, licenciada 1952, (mayo 2000).

55 Del Valle, T. et al. (2002), Modelos emergentes en los sistemas y las relaciones de género, Madrid, Narcea, p. 89.

56 La similitud de este perfil ideal con el que trazan las médicas que empezaron a ejercer treinta años después, es muy grande. También es similar la sensación de descontento por no encajar en el mismo, aunque entre las jóvenes tiene tintes más amargos: DELGADo SÁNCHEZ (2003), pp. 605-607. 
sus..., en hacerlo todo maravillosamente bien, en dar unas conferencias fantásticas (...) pero, a cambio de eso, la odiaban, era odiada, no la querían, «qué carácter tiene», pero claro, era la única forma de defenderse, claro, yo creo». ${ }^{57}$ familia...

«-El trabajo de investigación se daba más en el hombre que en la mujer, por la

- Es problema de tiempo, ¿entiendes?, y estudiar [las solteras] pueden estudiar mucho más que nosotras.

- La que es soltera pues le puede dedicar mucho tiempo, pero yo, con cuatro hijos, una consulta, un marido médico y de todo, hay muchas veces que tienes que poner la lavadora, que tienes que hacer la cena, hombre, y la vida no te da para tanto.» ${ }^{58}$.

"Yo he tenido que decidir en mi vida en un momento determinado si me iba a Francia a ser esposa o me quedaba aquí a ser profesional. Yo me he quedado aquí. Porque el ser esposa solo no me satisface. Entonces, yo admiro muchísimo a las mujeres casadas que han llevado una casa, que han criado unos hijos, y que encima se han dedicado a la profesión, porque veo que, a lo mejor yo lo que no hubiera podido es hacer las cosas que hacía, me hubiera tenido que conformar con una faceta o con dos facetas, no a pleno rendimiento, como yo lo he hecho. Pienso que eso es así»» ${ }^{59}$.

$\mathrm{P}: ¿$ ¿No casarse benefició su carrera investigadora? R: «Creo que sí, verdaderamente, porque lo que es la vida que llevo ahora no sé lo que me durará, ¿verdad?, pero es una vida demasiado fastidiada, ¡eh!, porque cuando llego a casa sigo y los domingos los días y las noches y, muy mal, porque no doy abasto» ${ }^{60}$.

Tanto las fuentes orales como las escritas permiten ver que las médicas ejercieron en los años sesenta y, especialmente, las que participaron activamente en la AEMM, se identifican a sí mismas de manera clara y rotunda como «mujeres-médicos» y que buscaron modelos diferentes a los dominantes para construir una identidad profesional común. En general, la conciencia de tener un estilo de práctica propio, que consideran femenino y valoran positivamente, así como unos intereses profesionales que nacían del hecho de ser mujeres, era habitual entre las profesionales que se iniciaron en la práctica a comienzos en los años 50 y 60 . En esa conciencia iba implícito un cierto orgullo, así como una aspiración de transformación y mejora de la práctica médica. Una dermatóloga, licenciada en 1952, relata así una de sus primeras experiencias laborales:

57 Entrevista OF.

58 Grupo operativo de antiguas socias de AEMM.

59 Entrevista PN, licenciada en 1954, (mayo 2000).

60 Entrevista MM, licenciada en 1957, (mayo 2000). 
«[en el año 1955] tuve que pasar una consulta, una sustitución, y ese señor, ese dermatólogo, ya me había hecho ir un día antes de casarme para que viera, y me dijo «mire, ahora va a ver usted como tiene que pasar visita» (era entonces muy joven, claro), bueno total que dice «sor (había una monja allí en el consultorio) traiga la silla» y la monja coge una silla y la pone así en el suelo. Entonces, «ique pase el primero!». Había dos o tres enfermos, y el enfermo se ponía detrás de la silla de pie y me dice «usted, cuando vengan los enfermos no los deje sentar, ahí detrás, las enfermedades de piel son todas muy contagiosas, así que no se le acerque ninguno», digo «bueno, bueno»; total cuando fui yo sola, yo poner aquí la silla, no, no, total que yo el enfermo aquí a mi ladito y, bueno, al otro ladito, empecé el mes con..., tenía dos o tres enfermos y cuando terminé tenía veintitantos. Mira, cuando llegó el y vio lo que yo había hecho me dijo que si seguía así que nunca más [...] Ve usted los hombres (risas). Las mujeres no somos capaces de hacer eso. Pero, bueno, para bien de la humanidad. Nosotras tenemos algunas virtudes. ¡Se imagina usted hacer eso!» ${ }^{61}$.

«- (...) los hombres, pues en aquella época pues salían [del quirófano] hablaban con los padres... Esa ambición que tienen los hombres a mi nunca, yo nunca me he pavoneado ni nada y he hecho las cosas, las he intentado hacer bien, pero los hombres, es verdad, que si he hecho esto, lo otro, necesitan..., yo no.

- Tienen una ambición

- Mucho, mucho, el salir a hablar con los padres sobre ..... y, así manchados de sangre y dándose importancia, yo esas cosas nunca las necesité $\rangle^{62}$.

«[La forma de ejercer] depende de la personalidad, de la cultura, de la inteligencia y creo que ahí ... [no hay diferencias de sexo] (...) Yo creo que yo tengo más interés por saber como va un ovario que como va una próstata (risas), ¿no? Yo creo que eso es instintivo» ${ }^{63}$.

Para concluir, las médicas españolas que ejercieron durante la década de los años sesenta, un momento de despegue de la participación de las mujeres en la medicina española, asumieron el discurso y las prácticas patriarcales de su tiempo como una forma, o una estrategia, de integración profesional. Sobre la base de experiencias y sentimientos ambivalentes, construyeron sólidas trayectorias e identidades profesionales que abrieron camino a las profesionales que vinieron después.

Esta investigación permite ejemplificar la potencialidad de las fuentes orales para incorporar a la historia de la profesión médica una perspectiva plural

61 Entrevista AL, socia, licenciada en 1952 (octubre 1999).

62 Grupo operativo de médicas licenciadas en los años sesenta, (mayo de 1999). Sobre la actitud corporal y el embodiment, o proceso de inscribir y representar a través del cuerpo la pertenencia social, CASELL (1998) lo analiza para el caso de las y los especialistas de cirugía.

63 Entrevista OF. 
sobre las formas de organización profesional y sobre los procesos de configuración y cambio de las prácticas y las identidades profesionales. Permite también mostrar su papel para incorporar y dar valor a la experiencia y a la subjetividad de los sujetos históricos. Se trata de fuentes únicas para conocer las formas de organización y asistencia médica en la España del siglo XX y para comprender los modelos que se están construyendo en el siglo XXI. Por todo ello, desarrollar un programa de recuperación y archivo de fuentes orales para la historia de las profesiones sanitarias, que incluyera relatos de varones y mujeres con experiencia en diferentes espacios de actividad, sería un paso trascendente en la historiografía médica española. 